ISSN 2693-2504

\title{
Standard operating Protocol (SOP) of Haematopoietic Stem Cells
}

\section{Enumeration}

Journal of Bioscience \& Biomedical Engineering

Research Article

Michael Halim*, Maureen Fatima Inuwa, Chidinma Angela Umenne and Kasali Abayomi Israel

University of Salford, MSc Biomedical Science, Greater Manchester, United Kingdom

\section{*Correspondence author}

Michael Halim

MSc Biomedical Science

University of Salford,

Greater Manchester

United Kingdom

Submitted : 14 Dec 2020 ; Published : 20 Jan 2021

$\begin{array}{ll}\text { Abbreviations } \\ \text { ACD : Acid Citrate Dextrose } \\ \text { BM }: \text { Bone Marrow } \\ \text { CPDA } & \text { : Citrate Phosphate Dextrose Adenine } \\ \text { FACS } & \text { : Florescent Activated Cell Sorting } \\ \text { HSC } & \text { : Hematopoietic Stem Cells } \\ \text { PB } & \text { : Peripheral Blood } \\ \text { PBSC } & \text { : peripheral blood stem cell } \\ \text { PPE } & \text { : Personal Protective Equipment } \\ \text { SOP } & \text { : Standard Operating Procedure } \\ \text { VBC } & \text { :Venous Blood collection }\end{array}$

Scope and Applicability

Human hematopoietic stem cells (HSCs) are obtained either from the bone marrow (BM), cord blood $(\mathrm{CB})$ or peripheral blood (PB). Transplantation of HSCs occurs following various conditions like high doses of chemotherapy, diseases like; leukaemia, lymphoma, congenital metabolic defects, immunedeficiency illnesses and myeloblastic syndromes.

Fluorescent activated cell sorting (FACS) is a specific type of flow cytometry used for analysing and sorting mixture of cells into various subpopulations using a specialized light scattering and fluorescent components of cells. These single test techniques characterize the cells when they are passed through a laser beam of light, this light has the capability to pick out and separate the cell population and the antibody reagent used has a known number of cytometric beads which is a standard for enumerating the cells [1].

The goal and objective of this SOP is to provide detailed instructions on the use of FACSVERSE ${ }^{\circledR}$ for enumeration of HSCs in $\mathrm{PB}, \mathrm{BM}$ or $\mathrm{CB}$ in a patient that needs transplantation or a donor and to facilitate training, accuracy and transfer of relevant information's needed for compliance while using or reading the SOP for enumeration of HSCs.
Accuracy of this analysis will assist the clinicians in the decision of blood transplant to a patient that needs it.

\section{Application}

The SOP is applied to all Clinical laboratory personnel's in NHS, Non clinical personnel's, health workers and clinical pathologies.

\section{Clinical association and potential diagnosis}

Haematopoiesis, is a life time process of blood production that occurs in a living organism by HSCs capable of selfrenewal and regeneration of diverse mature blood cells that are responsible for carrying oxygen, prevent bleeding, and fighting diseases. Clinically, successful cord blood, bone marrow or peripheral blood transplantation therapies occurs because of the ability for these tissues to regenerate in the host system. There are great limitations in finding suitable donors for HSC based treatment and transplantation. The clinical importance of determining precise and accurate numbers of this cells cannot be over emphasised.

Cell population of interest are characterised using the selection of fluorescent labelled antibodies that are specific to cell surface markers in HSCs enumeration. This cell surface markers are mostly glycoproteins referred to as clusters of differentiation (CD) markers which aid in differentiating cell subpopulations [2], Example CD44+, CD38-, CD38- CD90+ (thy-1), CD117 ${ }^{+}$ (c-kit), CD $34^{+}$and $\mathrm{CD} 45^{+} \mathrm{CD} 4 \mathrm{f}^{+}$and lineage markers (CD14, CD8-)

This report describes the SOP for enumeration of HSCs in PB in other to evaluate the patient need for transplantation, which is dependent on donor and recipients matching, graft-versushost response or leukaemia effect [3]. Accurately, enumerating HSCs will assist in the subsequent plans for apheresis collection [4].

Monoclonal Antibodies selection

Selection of appropriate antibody clone, entails selecting 
one that can retain a high specificity and validity of binding after conjugation with fluorochromes. A maximum of 6 color panels for the antibody estimation includes; Fluorescent Isothiocyanate (FITC) for detecting both isoforms and glycoforms in $\mathrm{CD}^{+} 5^{+}$antigens. Phycoerythrin (PE) a fluorescent dye for FACS analysis recommended for $\mathrm{CD} 34^{+}$ cell detection because, it has better demarcation between the positive and negative cells. Allophycocyanide (APC) a large protein with brightest fluorescent probe recommended for CD90 ${ }^{+}$. Peridinin-chlorophyll-protein complex (PerCP Cy5.5) is a tandem conjugate which links PerCP with cyanide dye recommended for $\mathrm{CD} 38^{+}$. PE-Cy7 is a tandem fluorochrome which consist of PE (R-phyco- erythrin) a protein excited by an Argon laser at $488 \mathrm{~nm}$ and also acts as an energy donor, coupled to the cyanine dye $\mathbf{C y} 7$ also recommended for $\mathrm{CD} 34^{+}$ detection [5].

\begin{tabular}{|l|l|l|l|l|l|l|l|}
\hline Fluorochromes & FITC & PE & APC & PerCp-Cy5.5 & PE-Cy7 & Lineage markers & Cell population tested \\
\hline Reagent $/$ markers & $\mathrm{CD} 38^{+}$ & $\mathrm{CD} 49^{+}$ & $\mathrm{CD} 117^{+}$ & CD34 & CD34 & CD14 & HSCs (BM, PB, CB) \\
\hline
\end{tabular}

Table 1: Antibody cocktail used for the 6 colour panels

\section{Safety considerations and specimen storage}

Specimen collection and storage:

- Peripheral blood should be aseptically collected by venipuncture into either $5-10 \mathrm{ml}$ tubes of ACD anticoagulated vacutainer and mix properly to avoid clotting or use CPDA stored blood via donation. (ACD or CPDA anticoagulant prolongs blood shelf life up to 28 days).

- All specimen should be properly labelled with date, time of collection and patient identifier, hospital reference number, date of birth with patient name and fore names.

- Clotted or lysed blood should not be used for this analysis.

- $\quad$ Specimen should be stored at room temperature $18-25^{\circ} \mathrm{C}$ or a constant temperature of $4^{\circ} \mathrm{C}$ and processed within the stipulated time (12hours maximum).

- WBC should be performed within six hours of collection.

Specimen transport:

- Specimen packaging and transport of peripheral blood, bone marrow, and apheresis or code blood should be according to the guidelines and regulation of the courier or postal services.

- The integrity of the specimen must be maintained and checked upon its receipt.

- In case of clots or lyses of specimen, a repeat collection should be requested.

- Specimen should not be exposed to light or extreme temperature because this can denature cells or cause hemolysis thereby affect measurement [6].

Safety precautions (Risk assessment):

Maintain all safety precautions while working in the laboratory, always adhere to the following safety practices;

1. Wear all personal protective equipment (PPE); Laboratory coat, hand gloves and safety glasses to protect the skin in case of blood or chemical spillage.

2. Sodium Azide, FITC, Phycoerythrin, APC and perCPCy5.5 has little amount of multiple chemicals used in antibody preparations therefore;

3. Avoid contact with skin, eyes and clothing and in case of any spillage, rinse immediately with large amount of water and seek medical aid.

4. Used hand gloves should be disposed in specified biohazardous bags.

5. Used pipette tips should be disposed carefully in bleach solution.

6. Clean all accidental spills using tissue paper and bleach spray and dispose in biohazard yellow labelled bin.

7. Workstation should be kept clean before and after use.

\section{Equipment, Supplies and reagents:}

- Gilson Pipette $(0.5 \mu 1-10 \mu 1$ and $100 \mu 1-1000 \mu 1)$

- $\quad$ Micro pipette tips

- $5 \mathrm{ml}$ Falcon tubes

- $1.5 \mathrm{ml}$ Eppendorf tube

- Timer

- Safety cabinet class II

- $\quad$ BD FACSVerse ${ }^{\circledR}$

- BD FACS hardware, software and gating device

- Vortex mixer

- Aspirator

- Computer monitor

- Centrifuge

- Water bath

- Biohazardous waste bag (yellow)

- Aluminum foil

- $\quad$ Latex gloves (medium, large, small)

- Sheath fluid

- Safety eye glass

- Tissue paper

- Bleach spray

- Test tube racks

- $1 \%$ Virkon

- Vacutainer and needles

- Cotton wool

- Tissue paper.

Reagent and monoclonal antibodies (MoAb)

- $\mathrm{CD}^{+} \quad$ PerCP-Cy5 (peridinin-chlorophyll-protein complex): BD 555823 (PE-Cy ${ }^{\mathrm{TM}} 5-$ Mouse Anti-human CD34).

- $\mathrm{CD}_{117^{+}}$APC (Allophycocyanide): BD 561118 (APC Mouse Anti-human CD117).

- Lineage markers; PE: BD 562530 (Phycoerythrin Human Mesenchymal Stem Cell Lineage Antibody Cocktail, with Isotype Control).

- CD38; FITC (Fluorescent Isothiocyanate): BD 555459 (FITC Mouse Anti-Human CD38).

- $\quad \mathrm{CD}_{49 \mathrm{f}^{+}}$(integrina 6); monoclonal antibody (ebioGoH3 (GoH3)) PE (Mouse, Human).

- $\quad$ CD90 : FITC Mouse IGg1, k isotype Ctrl (FC).

J B \& Bio Engine; $2021 \quad$ www.unisciencepub.com 
FACS buffers: stain cells extracellularly and used for cell resuspension before and after staining. Contains 1\% FCS and 0.05 sodium azide.

- $\quad 1 \mathrm{X}$ PBS $+1 \%$ FCS (dilution buffer for antibodies)

- BD FACS TMLysing solution 100ml- BD 349202 (BD FACS Lysis solution)

Specimen:

- Peripheral blood, Bone marrow or cord blood

- Donor blood collected in CPDA anti-coagulant.

\section{Procedures}

Antibody mix preparation

For staining and fixing the cells, pipette $0,5 \mathrm{ml}$ of whole blood into an identified Eppendorf (1.5ml) tube, add $200 \mu$ l of FACS dilution buffer and $2 \mu \mathrm{l}$ of each antibody (a-d) in the tube to lyse the red cells following staining.

Sample processing

- Label six (6) falcon tubes as from tube $1-6$

Tube 1 - Unstained control (is used to minimize the introduction of errors during experiment and to ensure the instrument is functioning properly also to control the background derived from auto fluorescence to set gating and voltage right), Tube 2 - FITC, Tube 3 - PE, Tube 4 - APC, Tube 5 - PerCP Cy5.5 and Tube 6 - Mixed stain (this contains all the recommended combination of primary antibody diluted in the right proportion).

- Add $0.5 \mathrm{ml}$ blood specimen in all 6 tube.

For each test use a new micropipette tip to add the right volume of well mixed anticoagulated blood to the base of each tube to ensure proper staining to obtain good result.

- $\quad$ Add $200 \mu 1$ of dilution buffer into unstained tube 1 .

- $\quad$ In FITC tube 2 add $2 \mu 1$ of reagent A -CD38

- $\quad$ In PE tube 3 add $2 \mu 1$ of reagent B- CD34 PE

- $\quad$ In APC tube 4 add $2 \mu 1$ of CD117 APC.

- $\quad$ In PerCP Cy5.5 tube 5 add $2 \mu 1$ of Lineage PerCP Cy5.5

- Into tube 6 containing mixed stain add $200 \mu 1$ of antibodies mix prepared at step 1

- Vortex tube carefully for 3 seconds to mix.

- Incubate tubes for 30 minutes in the dark or cover all tubes with aluminum foil at room temperature $20^{\circ} \mathrm{C}-25^{\circ} \mathrm{C}$ (exposure to light can cause white blood cell destruction)

- After the incubation period, add $1 \mathrm{ml}$ of $1 \mathrm{X}$ BD FACS Lysis buffer solution in all tubes, re-incubate further for 5 minutes at room temperature.

- Centrifuge tubes for 5 minutes at $1500 \mathrm{rpm}$ and discard/ aspirate supernatant carefully into a bleach solution (leave some residual fluids at the bottom not to distort the pallet during aspiration).

- Add $1 \mathrm{ml}$ of FACS lysis buffer in the pallet (with some visible red cells debris) and at room temperature incubate the tubes for 5 minutes.

- Centrifuge the tubes at 1500 rpm for 5 minutes.

- Discard the supernatant carefully in bleach solution

- After centrifugation, a reddish pallet should be observed.

- $\quad$ Pipette $500 \mu 1$ of PBS into all tubes and vortex or tab carefully for 3 seconds to resuspend the pallet.

- Cover all tubes with aluminum foil until FACS Acquisi- tion, analysis using the BD FACSVERSE ${ }^{\circledR}$ and should be within 24 hours after staining.

\section{Data record and storage}

- All result must be properly checked and validated before issuance.

- $\quad$ Result should properly be documented and reported as cell $/ \mu 1$ or cells $/ \mathrm{ml}$ accordingly.

- A standard operating procedure should be in place for trailing result sent by mail or faxed or telephone in case of misplacement, errors or conflict.

- Proper back-up system should be put in place in soft and hard copy format to avoid unforeseen circumstances.

- Primary data file, report forms and worksheet should be stored for a minimum of six month or the destruction of any record must be in accordance to agreed guidelines of the facility

\section{Data acquisition and analysis}

Data acquisition was by BD flow cytometry the percentage of cell population was determined by the six or eight colour stem cell enumerator kits (CD45, CD34, CD38, CD117, Lineage PE, PerCP-Cy5.5 and APC) following the setup of the machine. Using the formula below the population of cell in a specimen as performance of trucount should be calculated on the specimen to be tested to ascertain the accuracy and performance of the FACS cytometer expressed as cells/ $\mu$ l. For measuring trucount, 20ul of a properly labelled BD trucount tube are used without touching the pallet (Bdbiosciences.com, 2019).

Cell population $($ cells $/ \mu \mathrm{l})=\frac{\text { Number of cell count event }}{\text { Number of single beads }} \times$ BD Trucount Bead Conc. x Dilution Factor

Example: number of cell count: 50000, dilution factor: 200ul, beads counts: 25,000 , starting volume: $2 \mu 1$

Total cell population cells $/ \mu \mathrm{l}=\frac{50000}{25000} \times 200 \times 2=800 \mu \mathrm{l}$.

Therefore, total cell population was $800 \mu 1$.

The percentage calculated as $(\%$ of positive stained cells by total trucount cells/ $\mu$ l

from Result obtained (FACSVerse ${ }^{\circledR}$ ): Mixed solution $-0.21 \%$. Unstained sample $-0.25 \%$

Percentage of single cells $=\frac{0.21}{100} \times 800=1.68 \%$

\section{Gating}

Gating is used for cell subpopulation selection for further analysis. The optical information of the BD FACS cytometry fluorescence data called the forward (FSC)and side scatter (SSC) are obtained from an angle of emitted light from cells analysed which are detected by the specialized censor Figure $2 \mathrm{~A}$ and B. This is useful to distinguish between lymphocytes and granulocytes and the scatter plots are useful means of acquiring data from flow cytometry visualisation. The information's are plotted with their intensities as such, FSC as the X-axis and SSC as the Y-axis figure 2. The name of each surface marker and fluorochrome used are labelled on the plots e.g. CD45 FITC. 
Positive cells for both markers are located on the upper right quadrant and negative cell markers are on the bottom left [7,8].

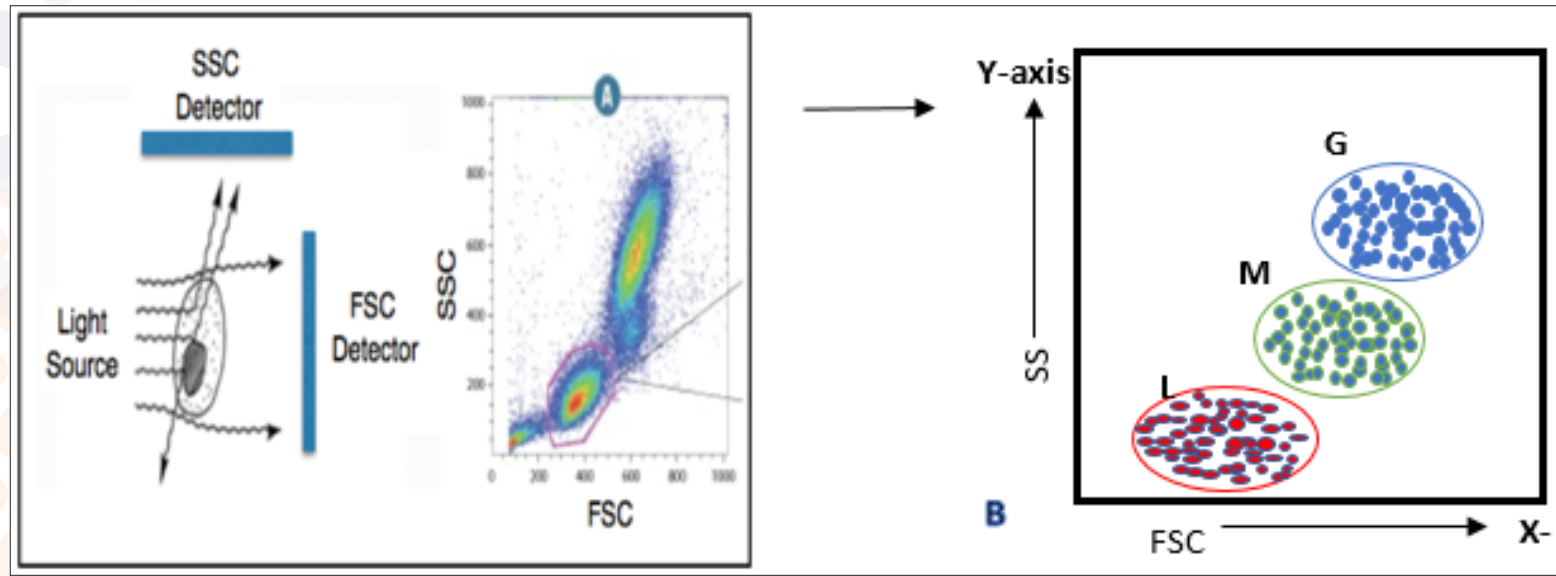

Figure 1: representative histogram showing HSC enumeration on the $\mathrm{x}$-axis, FSC- forward scatter and on the y-axis SSC- Side scatter FSC/SSC from a light source with gates subset indicating Florence cells from A and B: G- granulocytes. (M) Monocytes and (L) Lymphocytes.

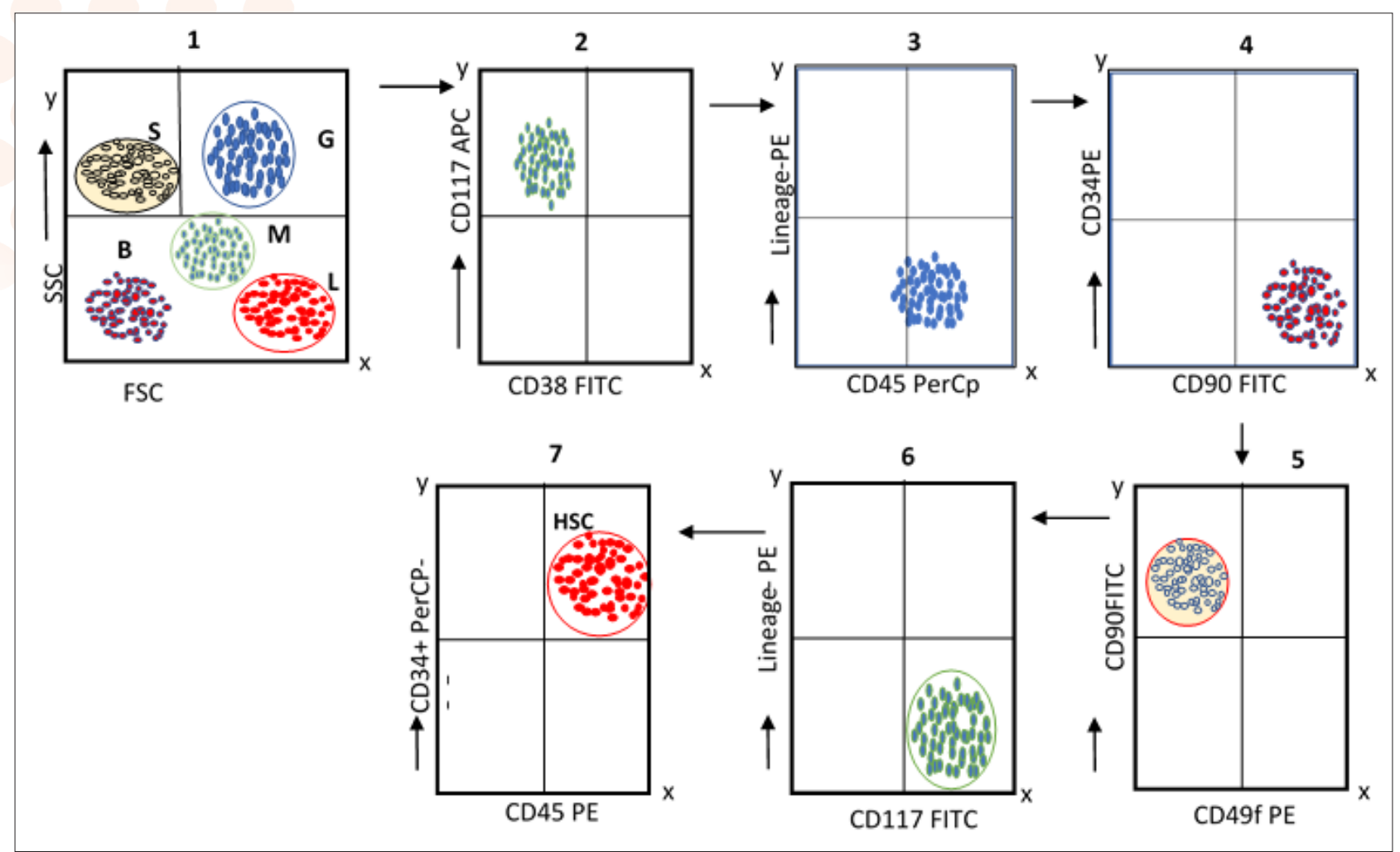

Figure 2: A representation of flow cytometry histograms of hematopoietic stem cell enumeration. (1) Multivariate histogram of FSC/SSC analysis with presumed gates. B blast cells. L lymphocytes. M monocytes. G granulocytes. S beads. (2) Coloured univariate plot of CD39/CD117. (3) CD45/lineage marker. (4) CD90/CD34. (5) CD49/CD90. (6) CD 117/Lineage. (7) CD45/ CD34. Cells enumerated by gating the positive cells for both markers.

\section{Quality assurance}

The research of Sanders and Karr, and Connolly and Wright defines quality assurance as the organized application of optimal processes in order to ensure the validity, reproducibility, and precise results of a test [9-11].

- All laboratory personnel's performing HSC enumeration test must be fully trained and certified to perform the test

- A process control is recommended to be used in order to monitor the performance of the reagent, stains, lysis and analysis carried out.

- Positive and negative controls should be run daily to ensure quality of result and when control specimens failed, they should be thoroughly checked and repeated [12].

- Control must be run if there is a change of reagent or lab personnel, calibration or service of equipment or if the test validity is questioned [1]. 
- Internal and external quality assurance should be undertaking, and all QA activities should be properly documented.

- Control data and results must be run and properly documented and verified before utilization e.g. positive control (BM or $\mathrm{CB}$ ) and negative control (normal venous $\mathrm{PB}$ ).

- Ensure daily, weekly or monthly maintenance and calibration of machines to keep them in optimal condition.

- Proper labeling and storage of samples in duplicates avoids the chance of losing large volume of specimens. This ensures that the quality of result does not undermine due to loss of sample.

Author's Contribution

Michael Halim, Maureen Fatima Inuwa, and Chidinma Angela Umenne contributed equally in this article.

\section{References}

1. Sutherland DR, Anderson L, Keeney M, Nayar R, ChinYee I (1996)The ISHAGE guidelines for CD34+ cell determination by flow cytometry. International society of hematotherapy and graft engineering. $J$ Hematother 5: 213-226.

2. Richard R. Jahan- Tigh, Caitriona Ryan, Gerlinde Obermoser and Kathryn Schwarzenberger. (2012) Flow cytometry. Journal of investigative dermatology 132: pp.1-6.

3. Tuthill, M. and Hatzimichael (2010) Hematopoietic stem cell transplantation. Stem Cells and Cloning: Advances and Applications p.105.

4. Murugesan, M., Nair, C., Nayanar, S. and Pentapati, K. (2019) Flow cytometric enumeration of CD34+ hematopoietic stem cells: A comparison between single- versus dual-platform methodology using the International Society of Hematotherapy and Graft Engineering protocol. Asian Journal of Transfusion Science, 13(1): p.43.

5. Berardi AC, Wang A, Levine JD, et al. (1995) Functional isolation and characterization of human hematopoietic stem cells. Science 267: 104-108.

6. Barnett, D., Janossy, G., Lubenko, A., Matutes, E., Newland, A. and Reilly, J. (1999) Guideline for the flow cytometric enumeration of CD34+ haematopoietic stem cells PREPARED BY THE CD34+ HAEMATOPOIETIC STEM CELL WORKING PARTY*. Clinical and Laboratory Haematology 21(5): 301-308.

7. Herzenberg, L., Tung, J., Moore, W., Herzenberg, L. and Parks, D. (2006) Interpreting flow cytometry data: a guide for the perplexed. Nature Immunology 7(7): 681-685.

8. Sanders, J.H., \& Karr, T. (2015) Improving ED specimen TAT using Lean Six Sigma. International journal of health care quality assurance 28(5): 428-440.

9. Downhousesoftware.files.wordpress.com. (2019) [online] Available at: https://downhousesoftware.files.wordpress. com/2016/07/screen-shot-2016-07-10-at-10-30-33-pm. png? $\mathrm{w}=768 \& \mathrm{~h}=387$

10. Bdbiosciences.com. (2019) [online] Available at: https:// www.bdbiosciences.com/ds/is/tds/23-3483.pdf [Accessed 29 Nov. 2019].
11. Connolly, D., \& Wright, F. (2017) The nursing quality indicator framework tool. International journal of health care quality assurance 30(7): 603-616.

12. Magnette, A., Chatelain, M., Chatelain, B., Ten Cate, H., \& Mullier, F. (2016) Pre-analytical issues in the haemostasis laboratory: guidance for the clinical laboratories. Thrombosis journal 14: 49. doi:10.1186/s12959-016-0123-z. 